\title{
Pediatric Cardiology Research in 1990: A Review of Abstracts Submitted to the Society for Pediatric Research, American Academy of Pediatrics, and American Heart Association Scientific Sessions
}

\author{
SAMUEL S. GIDDING, D. WOODROW BENSON, EDWARD B. CLARK, AND \\ ALBERT P. ROCCHINI \\ with the assistance of Sharon Hughes
}

\begin{abstract}
We assessed pediatric cardiology research by reviewing pediatric cardiology abstracts submitted in 1990 to the Society of Pediatric Research, American Academy of Pediatrics, and American Heart Association national meetings. Included were accepted and rejected studies. Abstracts were reviewed for disease being studied, methodology used to answer the research question, study design, and acceptance/rejection. Abstracts were analyzed from 123 institutions, 81 American and 42 foreign. Out of 423 abstracts, $307(72.6 \%)$ were clinical and the remainder were basic science investigations. Slightly more than half of the clinical submissions were related to congenital heart disease. Coronary artery disease and inflammatory diseases accounted for $12 \%$ of clinical submissions. Echocardiography, clinical outcome measures, and electrophysiology were the most common research methodologies. Almost $80 \%$ of basic science research was performed in normal tissues; animal physiology, fetal physiology, and cellular/biochemical studies were the most common methodologies. With regard to study design, half of the clinical studies were retrospective and only $6 \%$ were either prospective epidemiologic or prospective controlled intervention trials. For basic sciences, $38 \%$ of abstracts were descriptions of phenomena and $62 \%$ were hypothesis testing, with developmental hypotheses being most common. Acceptance rates favored higher quality study design. However, areas of greatest interest to cardiologists, congenital heart disease, cardiomyopathy, and electrophysiology, had poorer quality study design than did other areas. We have shown broad interest in pediatric cardiology research. However, clinical studies frequently were retrospective or had uncontrolled study designs. Basic science research was performed at a small number of institutions and emphasized either description of phenomena or developmental biology of normal tissues. (Pediatr Res 32: 10-16, 1992)
\end{abstract}

An analysis of the pediatric cardiology research effort is important to define resources, improve methodology, and identify new areas of inquiry. In addition, the assessment of the potential for future research must include an analysis of current research productivity and quality. Such an analysis should include measures of research quality, methodologies used for answering research questions, the contribution of clinical and basic science

Received November 25, 1991; accepted February 13, 1992.

Correspondence: Samuel S. Gidding, M.D., Division of Cardiology, Children's Memorial Hospital, 2300 Children's Plaza, Chicago, IL 60614. studies to total research effort, participation of the cardiology community, and the rewards for productivity.

We sought to study research in pediatric cardiology by reviewing pediatric cardiology abstracts submitted to national scientific meetings in 1990 . We characterized research reports by disease being studied, methodology used, study design, and acceptance rate. We found that clinical descriptive studies predominated and basic science studies were fewer and originated from a small group of institutions.

\section{MATERIALS AND METHODS}

Abstracts submitted to three national scientific meetings regularly attended by pediatric cardiologists were acquired. For the Society for Pediatric Research, all abstracts published in Pediatric Research (1) in the cardiology or neonatal cardiology sections were reviewed. For the American Academy of Pediatrics, all submissions were provided by the Scientific Program Director (D.W.B.); the subset of accepted abstracts has been published (2). For the American Heart Association, accepted abstracts were taken from those published in Circulation (3). Abstracts submitted but not accepted were obtained from reviewers with the permission of the Program Director and Council Chairman (A.P.R., E.B.C.). We estimated that $75-80 \%$ of all abstracts with content relevant to pediatric cardiology and submitted to these three meetings were available for review. Abstracts not available for review included those submitted to other pediatric subspecialty sessions of the Society for Pediatric Research $(n=3-5)$ and subsections of the American Heart Association where grading was not done by pediatric cardiologists (e.g. magnetic resonance imaging, basic science, community programs; $n=100$ 150 , number based on known acceptance rate for abstracts at this meeting and on estimate of pediatric abstracts presented in nonpediatric categories). This study does not include abstracts submitted to the American College of Cardiology Annual Meeting or abstracts with content relevant to pediatric cardiology submitted to meetings in other fields (e.g. genetics, teratology).

Each abstract was analyzed for the following variables: meeting submitted, institution of origin, country of origin, study design, basic or clinical science, methodology used to answer the research question, acceptance/rejection, and disease being studied. For clinical studies, methodology was defined as the technique used to answer the research question. For basic science studies, methodology was defined by the tool required to perform the investigation.

Pertinent subcategories for each analysis category are presented in the Appendix. Subcategory assignments were made by considering the study end points in each abstract. In general, assignments were easily made. When conflicts arose, the subcategory 
that was critical for evaluating the study end point was chosen. For example, a study of two-dimensional echocardiographic visualization of implantation of an atrial septal defect occlusion device was considered an echocardiographic rather than a catheterization study. Late follow-up of survivors of ventricular septal defect closure was considered as clinical rather than surgical because only clinical end points were considered in the outcome assessment.

Of particular importance was the study design end point, because this was the primary measure of research rigor. Subcategories were developed from a consideration of the typical types of clinical investigation reported in the medical literature (4). These include case reports, studies based on previously collected data (retrospective), studies acquiring data longitudinally based on a specific protocol (prospective), studies that make comparisons between two logically chosen groups (controlled), studies of population characteristics (epidemiologic, cross-sectional), and intervention trials in which biologic hypotheses, new drugs, or new treatments were evaluated. For basic science, these include descriptive studies, developmental biology, testing of biologic hypotheses, and assessments of disease models. The Appendix carefully describes the criteria used for applying each subcategory. All abstracts were assigned a category by one of the authors (S.S.G.).

Abstract grading: interobserver variability. A subset $(n=101)$ was reviewed by three authors (S.S.G. A.P.R., D.W.B.), and interobserver variability was assessed. This latter analysis provided a quality control measure for category assignment and allowed insight into the grading process. On abstracts reviewed by multiple observers, the majority opinion was given as the final assignment.

There was total agreement among graders in 28 cases, agreement by two observers in 62 , and no agreement in 11 . When there was no majority, consensus was established by first finding agreement for prospective/retrospective and then for overall study design (controlled/uncontrolled/epidemiologic). The most common disagreement among observers concerned whether studies were prospective or retrospective. No abstract received unanimity of opinion as to cross-sectional/epidemiologic study design. There was little disagreement with regard to assessment of basic science study design. The primary reviewer for all studies (S.S.G.) agreed with the consensus choice $83 \%$ of the time, thus validating use of these assessments for the remainder of the abstracts. The primary reviewer had an intraobserver variability of approximately $85-90 \%$, assessed by reviewing grades on submissions of the same abstract to two meetings.

Data were analyzed using the statistical package SPSS. Descriptive statistics and simple bivariate analyses described the data set. Adjustments were made for duplicate submissions $(n=27)$ and the absence of unaccepted abstracts $(n=37)$ where appropriate.

\section{RESULTS}

A total of 450 abstracts were reviewed. Of these, 27 were duplicate submissions, providing a total of 423 studies. They were received from 123 institutions, 81 in the United States and 42 in foreign countries. Figure 1 shows the number of abstracts submitted per institution. Of these, 307 of $423(72.6 \%)$ were clinical and the remainder were basic science investigations. Congenital heart disease was the most common disease in clinical submissions, whereas normal tissues were most common in basic science research. There were very few submissions in the areas of inflammatory disease, particular rheumatic fever, and coronary artery disease prevention (Table 1). Echocardiography was the most common methodology, followed by clinical studies and electrophysiology. Animal physiology, fetal physiology, and cellular/biochemical studies were the most common basic science methodologies (Table 1).

Study design. The frequency of the various study designs used

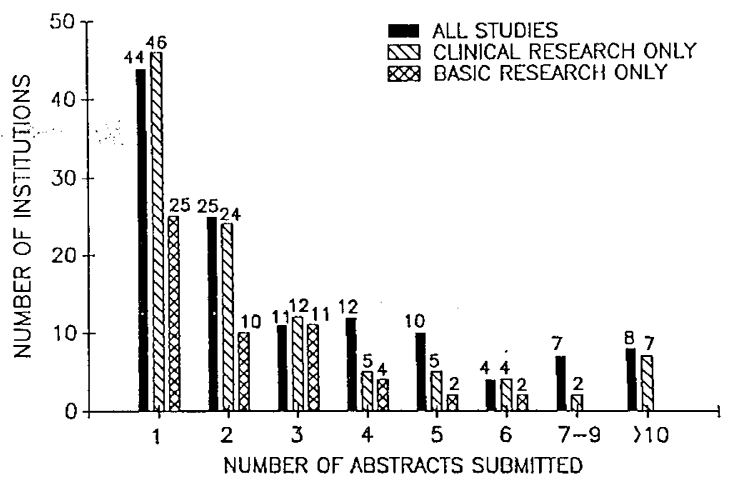

Fig. 1. The number of institutions submitting abstracts is shown. Sixty to $70 \%$ of institutions submitted one or two abstracts. The largest number of abstracts submitted by a single institution was 23 . Institutions submitting very large numbers of abstracts tended to submit mostly clinical studies. Twenty-three institutions submitted three or more basic science abstracts.

Table 1. Methodologies used in pediatric cardiology research and diseases or tissues studied

\begin{tabular}{|c|c|c|}
\hline Study type & Methodology & $\%$ \\
\hline \multirow[t]{9}{*}{ Clinical } & Echocardiography* & 20.6 \\
\hline & Clinical & 14.2 \\
\hline & Catheterization & 7.8 \\
\hline & Electrophysiology* & 6.1 \\
\hline & Biochemical/immune & 5.9 \\
\hline & Surgery* & 5.4 \\
\hline & New forms of imaging & 4.5 \\
\hline & Exercise & 3.4 \\
\hline & Other & 4.3 \\
\hline \multirow[t]{8}{*}{ Basic science } & Animal physiology & 8.7 \\
\hline & Biochemical/cellular & 6.0 \\
\hline & Fetal physiology & 5.0 \\
\hline & Arterial wall segment & 2.8 \\
\hline & Genetic/molecular biology & 2.6 \\
\hline & Other & 3.5 \\
\hline & & $\overline{100}$ \\
\hline & Diseases/tissues studied & \\
\hline \multirow[t]{8}{*}{ Clinical } & Congenital heart disease & 56.4 \\
\hline & Arrhythmia* & 10.1 \\
\hline & Coronary artery disease precursors & 7.8 \\
\hline & Cardiomyopathy/transplant & 7.2 \\
\hline & Normal children & 5.5 \\
\hline & Inflammatory diseases & 3.9 \\
\hline & Other & 9.1 \\
\hline & & $\overline{100}$ \\
\hline \multirow[t]{4}{*}{ Basic science } & Normal tissues & 77.6 \\
\hline & Congenital heart disease & 11.2 \\
\hline & Other & 11.2 \\
\hline & & $\frac{12 .}{100}$ \\
\hline
\end{tabular}

* May be slightly underestimated because of missing abstracts.

for clinical and basic science investigations are shown in Table 2. Approximately one half of the clinical studies were retrospective. About one third of the studies used a control group. Less than $6 \%$ of clinical studies were controlled-intervention trials or prospective epidemiologic investigations.

For the 116 basic science abstracts, $38 \%$ of abstracts were descriptions of phenomena and $62 \%$ were hypothesis testing. Developmental hypotheses (e.g. the fetus or neonate behaves differently from the adult) were the most common hypotheses used. Testing of biologic hypotheses, generally in normal tissues, accounted for $20 \%$ of submissions. Research with disease models accounted for $16 \%(n=19)$ of basic science abstracts submitted, 
Table 2. Study design frequency*

\begin{tabular}{lrr}
\multicolumn{1}{c}{ Study design } & $n$ & $\%$ \\
\hline Clinical & 20 & 6.7 \\
Case report & 101 & 33.9 \\
Retrospective (cases only) & 24 & 8.1 \\
Retrospective (case control) & 7 & 2.3 \\
Retrospective (cross-sectional) & 73 & 24.5 \\
Prospective (cases only) & 56 & 18.8 \\
Prospective (case control) & 6 & 2.0 \\
Prospective (randomized intervention) & 11 & 3.7 \\
Prospective (cross-sectional/epidemio- & - & \\
$\quad$ logic) & 298 & 100 \\
& & \\
Basic science & 44 & 38.3 \\
Description of a phenomenon & 28 & 24.3 \\
Developmental biology & 24 & 20.9 \\
Hypothesis testing & 19 & 16.5 \\
Disease model & 115 & 100 \\
\hline
\end{tabular}

${ }^{*}$ See Appendix for description of study designs.

and, of these, nine of 19 involved normal animals or tissues modified to create a disease state and the remainder involved animals or tissues with a specific abnormality.

Figure 2 describes clinical study design by the disease being studied. There is significant variation by disease type. Work done with coronary artery disease precursors and in normal children had the highest percentage of prospective controlled or epidemiologic submissions, whereas studies of arrhythmias, congenital heart disease, and transplant/cardiomyopathy had the most retrospective studies.

Study design varied widely by clinical research methodology. Figure $3 A$ presents the types of study design for specific research methodologies. Clinical, catheterization, electrophysiologic, and surgical reports were most likely to apply retrospective and uncontrolled study designs. Echocardiography and biochemical/ immunologic studies were most likely to use prospective study designs. For the basic sciences, there was also a significant variation in study design by research methodology. Figure $3 B$ shows the study designs for those methodologies with greater than 10 submissions. Genetics/molecular biologic, cellular/biochemical, and isolated arterial preparations had the most submissions testing specific hypotheses.

By institution, 49 of 105 (47\%) submitting clinical abstracts had at least one study with a prospective controlled design. Basic science studies were submitted from 54 of 123 centers (44\%).

Interaction of methodology used and disease studied. There were significant differences in research methodology used by type of disease in clinical investigations. Congenital heart disease abstracts were dominated by echocardiography $(n=49)$, clinical outcomes $(n=32)$, catheterization $(n=23)$, surgery $(n=21)$, and newer forms of imaging $(n=14)$. Preventive cardiology was dominated by biochemical end points (12 of 23). Cardiomyopathy/transplant used echocardiography (11 of 24) and clinical end points (7 of 24). Normal subjects were usually studied by echocardiography. Arrhythmias were usually studied by electrophysiologic techniques or clinical end points.

Comparison of American and foreign submissions. There were significant differences between American and foreign submissions. For clinical research, study designs were similar; however, foreign submissions emphasized congenital heart disease $(66 \%$ foreign versus $44 \%$ American) over other diseases, particularly coronary artery disease (3\% foreign versus $9 \%$ American). Cardiac catheterization was more commonly used by foreign countries in clinical investigation (19\% foreign versus $8 \%$ American) than echocardiography (23\% foreign versus $30 \%$ American). Basic science studies from foreign countries emphasized animal and fetal physiology (85\% foreign versus $44 \%$ American). Most

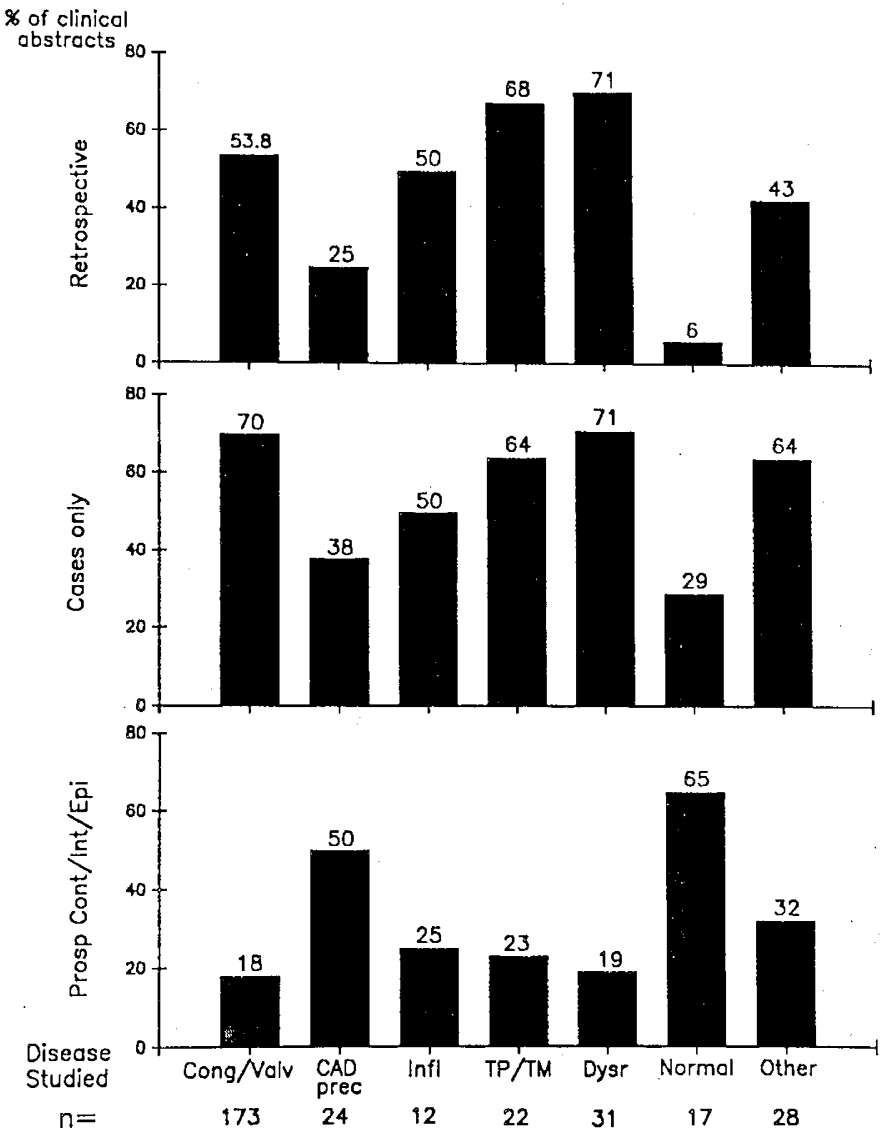

Fig. 2. Study designs of abstracts submitted for particular diseases are shown. The upper panel shows the percentage of submissions that used retrospective designs: case reports, retrospective cases only, retrospective case control, and retrospective cross-sectional. The middle panel shows the percentage of submissions that did not have control groups: case reports, retrospective cases only, and prospective cases only. The lower panel shows the percentage of studies that used prospective controlled, prospective controlled intervention, and prospective epidemiologic study designs. CAD prec, coronary artery disease precursors; Cong/Valv, congenital/valvular heart disease; Dysr, dysrhythmia; Infl, inflammatory; TP/TM, transplant/cardiomyopathy.

foreign submissions (80\%) went to the American Heart Association.

Acceptance/rejection. Basic science acceptance rates were much higher than clinical acceptance rates [70 of $116(60.3 \%)$ versus 83 of $297(27.9 \%)]$. Acceptance rates by study design are shown in Table 3 and suggest that, overall, acceptance rate is determined by study design, inasmuch as prospective and epidemiologic studies had higher acceptance rates. However, when clinical studies were analyzed either by methodology used or by disease studied, there was a weaker relationship. Figure 4 graphs the acceptance rates by methodology and by disease type for clinical studies. These graphs suggest that for many categories study design determines likelihood of acceptance; however, catheterization studies had higher acceptance rates than their study designs would warrant. Acceptance rates were relatively constant across disease types despite a wide variation in study design by disease type.

Differences among meetings. There were many differences among the meetings with regard to study end points. The Society for Pediatric Research (SPR) attracted the highest percentage of basic science abstracts [46\% SPR versus $21 \%$ American Heart Association (AHA) versus 19\% American Academy of Pediatrics (AAP)], and, with the American Academy of Pediatrics, accepted the highest percentage of basic science studies (69\% SPR versus $68 \%$ AAP versus $47 \%$ AHA). For clinical studies, the acceptance 


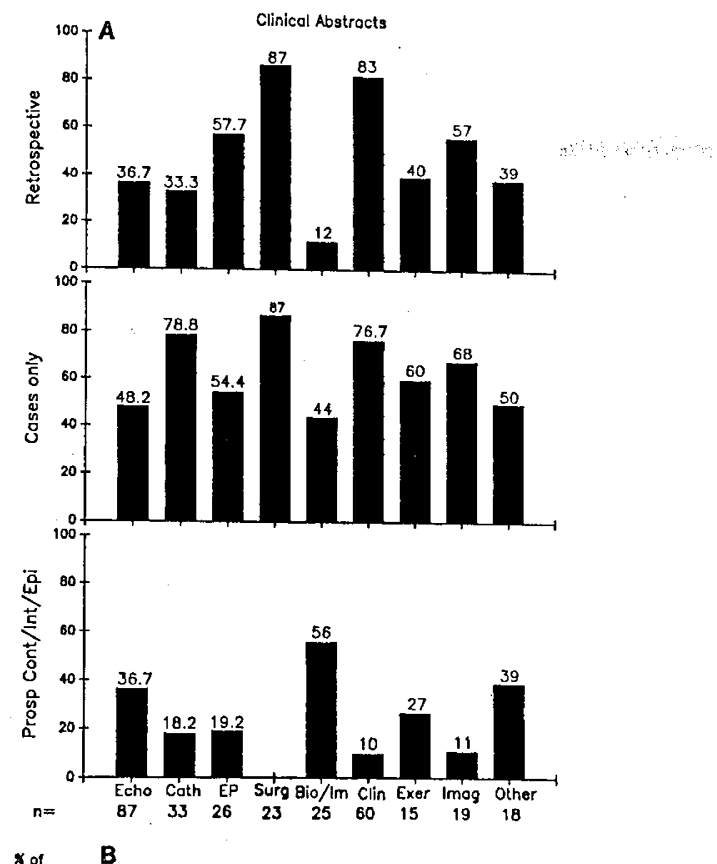

Table 3. Acceptance rates by study design

\begin{tabular}{lrc}
\hline \multicolumn{1}{c}{ Study design } & $n$ & $\begin{array}{c}\text { Acceptance } \\
\text { rate (\%) }\end{array}$ \\
\hline Clinical & & \\
Case report & 20 & 15 \\
Retrospective (cases only) & 101 & 19 \\
Retrospective (case control) & 24 & 13 \\
Retrospective (cross-sectional) & 7 & 56 \\
Prospective (cases only) & 73 & 38 \\
Prospective (case control) & 56 & 36 \\
Prospective (intervention trials) & 6 & 50 \\
Prospective (cross-sectional) & 11 & 55 \\
Basic science & & \\
Descriptive & 44 & 55 \\
Developmental & 28 & 75 \\
Biologic hypotheses & 24 & 67 \\
Disease model & 19 & 47 \\
\hline
\end{tabular}
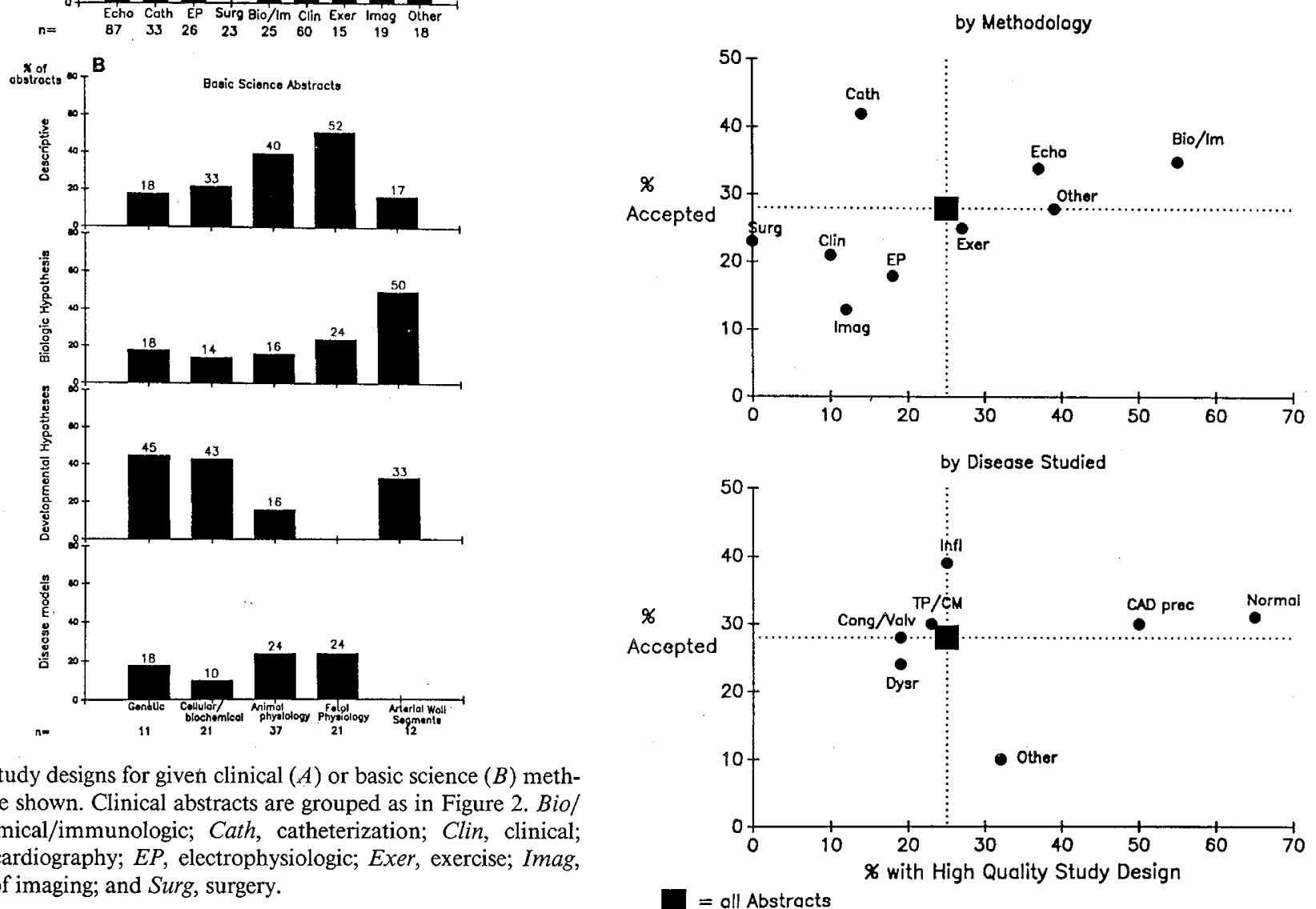

Fig. 3. Study designs for given clinical $(A)$ or basic science $(B)$ methodologies are shown. Clinical abstracts are grouped as in Figure 2. Bio/ Im, biochemical/immunologic; Cath, catheterization; Clin, clinical; Echo, echocardiography; EP, electrophysiologic; Exer, exercise; Imag, new forms of imaging; and Surg, surgery.

rates were similar ( $26 \%$ SPR versus $27 \%$ AAP versus $31 \%$ AHA); however, the SPR received the highest percentage of prospective clinical studies (57\% SPR versus $21 \%$ AHA versus $25 \%$ AAP). Only the American Heart Association received a significant number of contributions for coronary artery disease prevention. The Society for Pediatric Research attracted a lower percentage of surgical studies and higher percentages of echocardiographic and animal physiology studies.

\section{DISCUSSION}

We have described the current research output of pediatric cardiologists by assessment of abstracts submitted to three national meetings. Measures used to assess abstracts included measures of quality (study design), methodologies used to answer

Fig. 4. The graphs show the relationship between acceptance rate and quality of study design. The upper panel groups abstracts by the research methodology and the lower panel groups abstracts by the disease studied. High quality study design was one of the following: prospective controlled, prospective randomized intervention, or prospective cross-sectional/epidemiologic. The black square shows the percentage of all abstracts submitted that were accepted and the percentage of all abstracts that had high quality study design. Abstract groups in the left lower quadrant had poorer study designs and poorer acceptance rates, and those in the right upper quadrant had better study design and higher acceptance rates. Abstract groups in the left upper quadrant had poor study designs with high acceptance rates, and those in the right lower quadrant had better methodology but lower acceptance rates. See text for further discussion. Abbreviations are the same as those used in Figures 2 and 3. TP/CM, transplant/cardiomyopathies. 
research questions, institutional participation, diseases under study, and the reward system (acceptance rates, interobserver variability for assessment of study design). Clinical submissions were much more common than basic science submissions. Basic science studies emphasized description of phenomena or developmental biology; however, there were a significant number of studies devoted to biologic hypothesis testing and disease models. A broad range of methodologies was used to answer research questions.

There were many positive findings. Most encouraging was the broad level of interest in research, with 81 U.S. institutions. submitting material to at least one meeting, as well as the attraction of significant numbers of foreign institutions to these meetings. Overall, $46 \%$ of institutions submitted some basic science material and $44 \%$ submitted at least one study with a prospective controlled study design. The abstract review process rewards clinical investigators who use prospective study design with higher acceptance rates. Higher acceptance rates were also observed for basic science investigation, although it was disconcerting that the very best clinical designs did not achieve acceptance rates comparable to basic science studies.

There are examples of controlled interventional investigations conducted by pediatric cardiologists and larger collaborative studies that have yielded important results for the practice of pediatric cardiology $(5-10)$. These studies have added or evaluated new therapies in the field, assessed physiology underlying clinical observations, and uncovered serious problems unrecognized by smaller trials. Although many current abstract submissions demonstrate the feasibility of using new therapies or technologies, using a retrospective or cases only format, they less often take the next step, employing higher quality study designs to answer key physiologic or clinical questions occasioned by the use of these new technologies.

Descriptive clinical studies have been critical to the development of pediatric cardiology. The first description of atrial balloon septostomy was a case report (11). The natural histories of many disease states were first elucidated in retrospective series (12). However, as the field matures, descriptive studies without follow-up trials appropriately controlled may not provide sufficient information about the natural history of a disease or the value of a new treatment or procedure. As financial restraints on clinical care grow, analysis of clinical outcome and cost effectiveness in large trials become essential elements in defining the distribution of health care resources. These studies have ethical implications, because physician use of new and high-priced technology is coming under closer scrutiny.

Because neither the writing content of abstracts nor that of presentations was assessed, we could not criticize the quality of individual abstracts. However, several trends in the data suggest areas for improvement. There was a paucity of prospective, controlled interventions and epidemiologic investigations. Many of the areas of greatest interest to clinical cardiologists, e.g. congenital heart disease, arrhythmias, and cardiomyopathy, were dominated by retrospective and uncontrolled investigations. A goal of pediatric cardiology research should be to increase the number of prospective studies and provide better controls for studies that must be performed retrospectively.

We believe our analysis has implications for the role of pediatric cardiologists in basic research. The number of basic science papers was small, they came from fewer institutions than did clinical papers, and they were dominated by studies in normal tissues rather than in disease models, where most clinical problems reside. Either pediatric cardiologists are submitting their basic science reports to other meetings or fewer pediatric cardiologists are pursuing basic studies. This paucity of investigators may adversely affect the progress of basic science related to children's heart health. A recent survey found only 16 pediatric cardiologists who assigned more than $20 \%$ of their time to basic investigation (Moller JH, personal communication). The lack of mentors and role models adversely influences recruitment of pediatric cardiologists into both basic and clinical research. This may help explain the small number of basic science submissions. A small pool of role models will not attract an adequate number of trainees. In return, basic investigation becomes less credible in the eyes of both trainees and faculty, further contracting the field.

It was difficult to evaluate several key areas of abstract submission given the limitations on the ability to collect reliable data. The quality of abstract writing was not assessed, although it was clear from attempting to assign categories that a lack of clear presentation created ambiguity for reviewers and blunted the effect of the presentations. This may account for part of the intraobserver and interobserver variability with regard to the prospective/retrospective nature of the abstracts. Cross-sectional epidemiologic studies produced disagreements among observers as to their true study design because there was often no true hypothesis statement at the beginning of the abstract.

A subtle bias against surgical and catheterization submissions was placed in the study design analysis, a bias created by the ethical difficulty of providing appropriate controls. Many of these studies must be retrospective. Conversely, studies of interventional catheterization, echocardiography, electrophysiology, and exercise often are evaluating new technology and procedures and are therefore evaluating protocols that are not standard clinical practice. Consequently, these can be considered prospective investigations and they may have internal controls. These abstracts have "better" study designs, but they may be no more or less descriptive than many surgical or catheterization studies.

Basic science studies produced few difficulties in interpretation among graders. This is of interest because there is always a concern about the reviewer's familiarity with the subject matter of many of these submissions. Although it may be difficult for unfamiliar observers to judge the contribution of a particular work to its field, it was relatively easy to assess the thought process behind a particular project.

A bias may exist toward acceptance of some studies over others because of the way scientific sessions are planned. A specific time allotment may be made by the program committee for certain types of abstracts (e.g. interventional catheterization, echocardiography, Young Investigator Award), thus guaranteeing that a specific number of abstracts will be accepted independent of quality in those areas. Newer methodologies may be favored over older methodologies because of interest rather than research quality. This may help explain the relatively constant acceptance rate across disease types and the variation in acceptance rates by methodology seen in Figure 4. Different meetings attract different types of abstracts. For example, surgical, preventive cardiology, and foreign submissions are more likely to go to the American Heart Association because of the large audience outside of pediatric cardiology as well as the presence of more slots for presentations.

A major goal of this study was to provide a snapshot of the spontaneous research output of pediatric cardiologists. Abstracts provide good material for this goal. However, if an overview of the entire research process were sought, a review of published papers and grant applications would be required.

Increasing financial pressures and clinical service demands placed on academic centers may potentially compromise medical research, particularly that research carried out by physicians. Pediatric cardiology, a field with large clinical service needs driven by new technologic developments, is vulnerable to these pressures. It is important for overviews such as this to influence investigators to use rigorous study designs in the evaluation of new methodologies, to encourage collaborative studies, to identify key areas for investigation, and to encourage extramural funding agencies to appropriate funds to allow quality investigation in key areas. This study is useful in defining the scholarly output of the pediatric cardiology community in the 1989-1990 academic year. However, we note that this paper is a noncon- 
trolled, descriptive study and that it suffers from all of the weaknesses of that study design.

\section{REFERENCES}

1. Pediatr Res 1990;27:15A-27A, 232A-238A(abstr)

2. Am J Cardiol 1990;66:518-527(abstr)

3. Circulation 1990;82:III-19-20, 151-154, 186-188, 222-224, 352-354, 402$404,437-439,490-492,501-502,582-584,715-717$ (abstr)

4. Monson RR 1990 Occupational Epidemiology, 2nd Ed. CRC Press, Boca Raton, FL, pp 15-61

5. The Cardiac Arrhythmia Suppression Trial (CAST) Investigators 1989 Preliminary report: effect of encainide and flecainide on mortality in a randomized trial of arrhythmia suppression after myocardial infarction. $N$ Engl $J$ Med 321:406-412

6. Ferencz C, Rubin JD, McCarter RJ, Brenner JT, Neill CA, Perry LW, Hepner SI, Downing JW 1985 Congenital heart disease: prevalence of live birthThe Baltimore-Washington Infant Study. Am J Epidemiol 121:31-35

7. Lauer RM, Lee J, Clarke WR 1988 Factors affecting the relationship between childhood and adult cholesterol levels: The Muscatine Study. Pediatrics 82:309-318

8. Gidding SS, Rocchini AP, Beekman R, Szpunar CA, Moorehead C, Behrend D, Rosenthal A 1985 Therapeutic effect of propranolol on paradoxical hypertension after repair of coarctation of the aorta. N Engl J Med 312:1224 1228

9. Newburger JW, Takahashi M, Burns JC, Beiser AS, Chung KJ, Duffy CE, Glode MP, Mason WH, Reddy V, Sanders SP, Shulman ST, Wiggins JW, Hicks RV, Fulton DR, Lewis AB, Leung DYM, Colton T, Rosen FS, Melish ME 1986 The treatment of Kawasaki syndrome with intravenous gamma globulin. N Engl J Med 315:341-347

10. Castaneda AR, Trusler GA, Paul MH, Blackstone EH, Kirklin JW 1988 The early results of treatment of simple transposition in the current era. J Thorac Cardiovasc Surg 95:14-27

11. Rashkind WJ, Miller WW 1966 Creation of an atrial septal defect without thoracotomy. A palliative approach to complete transposition of the great arteries. JAMA 196:991-992

12. Nadas AS 1977 Pulmonary stenosis, aortic stenosis, ventricular septal defect: clinical course and indirect assessment. Circulation 56(suppl):I1-I87

\section{APPENDIX}

\section{SUBCATEGORIES FOR ABSTRACT CLASSIFICATION}

\section{Country of origin}

Disease being studies answer the research question

\section{Clinical}

\section{Basic science}

$\therefore$

\section{Clinical Studies}

STUDY DESIGN CATEGORIES:

TYPE AND DESCRIPTION

Case report: Studies that include less than five subjects; included in this code are genetic studies that involve one family only.

Retrospective cases only: Studies in which data were collected retrospectively; results are not compared with a well-defined control group; some comparisons may be made among arbitrarily defined subgroups.

Retrospective case control: Data is collected by review of patient material; however, the review is directed specifically toward a comparison, e.g., patients who have had the Fontan operation, with a comparison made between tricuspid atresia and other types of single ventricle. Individual subjects may serve as their own controls if the comparison is logical with regard to the study hypothesis, e.g., epicardial versus transesophageal imaging of the same patient.

Retrospective cross-sectional: Data will be collected retrospectively but will be analyzed on a cross-sectional basis (regression analysis is performed appropriately for assessment of study end points); generally a large number of cases should be included in the abstract.

Prospective cases only: Studies that appear to have been done prospectively. Only subjects with a particular disease or intervention are included in the analysis. No meaningful comparison to a control group is made, and/or no obvious attempt to include a control group in the analysis is made. For many studies, it will be very difficult to distinguish between retrospective and prospective design. The following criteria can be used to discriminate a prospective design: the procedure or investigative technique is not part of standard therapy, every patient in the described treatment group receives exactly the same intervention or treatment, and there is reasonable evidence that all candidates for the treatment or intervention from a specific center have been included in the analysis. This definition may lead to a selection bias against surgical and catheterization studies, which are more easily identified as retrospective. Exercise, electrophysiologic, biochemical, and echocardiographic studies can appear to be prospective because some of the data collected are novel; however, they may actually be retrospective.

Prospective case control: These studies include the prospective collection of data and a clearly defined control group that is pertinent to the analysis of the hypothesis; subjects can serve as their own controls if appropriate for the hypothesis. Abstracts in this group should have a hypothesis clearly articulated. Studies in which multiple, different assessments of a similar phenomenon are made in an individual patient count in this category (e.g. echocardiography versus catheterization gradient).

Prospective randomized intervention: These studies are prospective and include two groups of relatively equal composition; one receives a treatment or intervention and the other does not.

Prospective cross-sectional/epidemiologic: These are prospective trials in large populations testing specific hypotheses, often requiring sophisticated statistical analysis. 
Basic Science

Description of a phenomenon, no definite hypothesis: These are studies in which a specific drug or intervention is tested, and the results describe what happened in an experimental preparation.

Developmental biology: Comparisons are made at different stages of development. Comparisons can be made among various stages of fetal life, neonatal life, childhood, and adulthood.
Hypothesis testing: The authors clearly state a hypothesis and apply appropriate interventions to their experimental preparation to elucidate that hypothesis, and the study is appropriately controlled.

Disease model: The research is done on tissues that are not normal (e.g. turkey cardiomyopathy), or the studies are done on a model constructed from normal tissues (e.g. a fetal lamb with an inferior vena cava-left atrial shunt).

\section{Announcement}

\section{NIH Consensus Panel Issues Report: Consensus Development Conference on Acoustic Neuroma}

A National Institutes of Health (NIH) consensus development statement on acoustic neuroma may be obtained from the NIH Office of Medical Applications and Research.

The report was prepared by a panel of experts who considered scientific evidence presented at a Consensus Development Conference at NIH. It contains the recommendations and conclusions concerning acoustic neuroma. Free, single copies of the consensus statement on acoustic neuroma may be obtained from William H. Hall, Director of Communcations, Office of Medical Applications and Research, National Institutes of Health, Building 1, Room 259, Bethesda, MD 20892, (301) 496-1143. 